\title{
Hippocampus is required for paired associate memory with neither delay nor trial uniqueness
}

\author{
Jinah Yoon, Yeran Seo, Jangjin Kim, and Inah Lee ${ }^{1}$ \\ Department of Brain and Cognitive Sciences, Seoul National University, Gwanak-gu, Seoul 151-742, Korea
}

\begin{abstract}
Cued retrieval of memory is typically examined with delay when testing hippocampal functions, as in delayed matching-tosample tasks. Equally emphasized in the literature, on the other hand, is the hippocampal involvement in making arbitrary associations. Paired associate memory tasks are widely used for examining this function. However, the two variables (i.e., delay and paired association) were often mixed in paired associate tasks, and this makes it difficult to localize the cognitive source of deficits with hippocampal perturbation. Specifically, a few studies have recently shown that rats can learn arbitrary paired associations between certain locations and nonspatial items (e.g., object or flavor) and later can retrieve the paired location when cued by the item remotely. Such tasks involve both (1) delay between sampling the cue and retrieving the target location and (2) arbitrary association between the cueing object and its paired location. Here, we tested whether delay was necessary in a cued paired associate task by using a task in which no delay existed between object cueing and the choice of its paired associate. Moreover, fixed associative relationships between the cueing objects and their paired locations were repeatedly used, thus involving no trial-unique association. Nevertheless, inactivations of the dorsal hippocampus with muscimol severely disrupted retrieval of paired associates, whereas the same manipulations did not affect discriminating individual objects or locations. The results powerfully demonstrate that the hippocampus is inherently required for retrieving paired associations between objects and places, and that delay and trial uniqueness of the paired associates are not necessarily required.
\end{abstract}

[Supplemental material is available for this article.]

Animal models for testing episodic memory have been particularly useful for studying a selective contribution of the hippocampus and its neural mechanisms. One of the animal models in the literature involves teaching paired associative relationships between mnemonic items and then testing the retrieval of a learned item when cued by its paired associate (Bunsey and Eichenbaum 1993; Day et al. 2003; Kesner et al. 2005). Recently, Day et al. (2003) proposed an impressive rodent version of cued paired associate task, in which rats formed paired associate memory between a particular flavor and its paired associate location. Specifically, in the task, the rat should learn flavor-place paired association during spatial navigation in a sample phase. In the retrieval phase, the rat was cued with one of the flavors learned during the sample phase and was required to navigate to its paired associate place. Compared with other paired associate tasks (Cho and Kesner 1995; Bunsey and Eichenbaum 1996; Lee and Solivan 2008), animals must learn a trial-unique paired association in a single trial in this task. Furthermore, the rat was cued in the start box (thus, away from its original sampling location) during the test phase. Importantly, this feature of the task imposed a temporal gap, during which the cue information must be maintained during the search period after exiting the start box. All of the above features of the task appear to create a similar situation, in which episodic memory is formed and retrieved in humans. Kesner et al. (2008) also used a similar paradigm and reported that the CA3 subfield of the hippocampus is essential in this type of task.

The cued paired associate memory task with the delay mentioned above may depend upon several cognitive processes. First

\footnotetext{
'Corresponding author.
}

E-mail inahlee@snu.ac.kr.

Article is online at http://www.learnmem.org/cgi/doi/10.1101/lm.024554.111. of all, during the sample phase, a stable and discrete association between paired associates (e.g., nonspatial item and its location) should be formed based on a single exposure. In addition, during the retrieval phase, the representation of a cueing item should be maintained during the delay, as the animal tries to find its paired associate location. Finally, the animal must navigate an open space to locate the target place for obtaining a reward. It still needs to be determined whether all of these task components are necessary to recruit the hippocampal function maximally in a cued paired associate memory task. In order to test whether these conditions are necessary, we tested rats in a simpler task situation, where a minimal number of object-place paired associates were repeatedly used (thus, no trial uniqueness involved). Furthermore, the cueing object was always presented with candidate spatial locations to remove working memory demand in usual delayed-memory retrieval paradigms (Day et al. 2003; Kesner et al. 2005). Also, there was no need for spatial navigation in a large space in our task. Nevertheless, we report that inactivation of the dorsal hippocampus with muscimol (GABA-A receptor agonist) severely disrupted object-cued paired associate memory. Our results strongly suggest that the associative link itself (between item and place) becomes unavailable without normal hippocampal function in a spatial paired associate task.

\section{Results}

Twenty Long-Evans rats (270-330 g) were trained in an objectcued paired associate memory task in a modified linear track surrounded by distinct extramaze cues (Fig. 1A). In this task, the rat came out of the start box at one end of the track and approached a choice platform at the other end of the track. In the choice platform, the rat encountered a cueing object (either an egg-shaped 
A
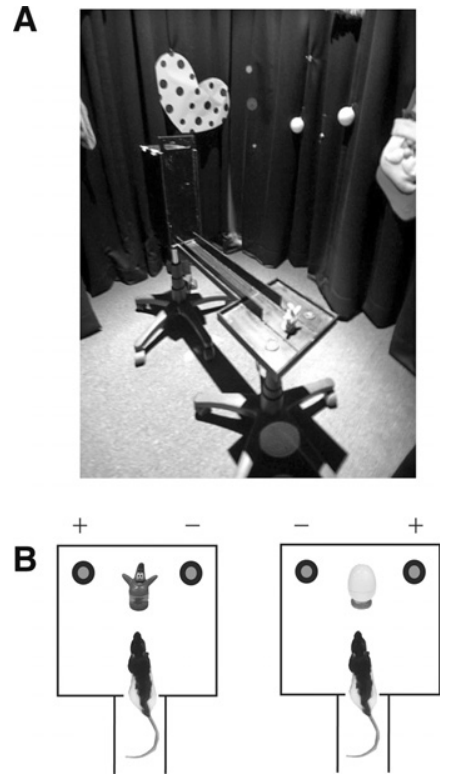

Figure 1. Behavioral testing room and the object-cued paired associate task. (A) Behavioral testing setup. In a circular curtained room with several visually distinctive cues presented along the curtains, a linear track with a choice platform at its end was placed in the center. A start box was located at the other end of the track. (B) Behavioral testing paradigm. In the choice platform, one of two different objects was presented in between two possible reward-containing places (denoted by + ) that were covered by identical metal discs. The availability of food reward in the food well was determined by the identity of the cueing object. For example, when a cartoon figure was presented in between the food wells, the rat was required to displace the disc over the left food well, whereas the disc on the right food well needed to be removed when an egg-shaped object was presented as a cue.

plastic toy or a cartoon figure as shown in Fig. 1B). The task was to choose the left or right food well (covered with identical washers) associated with the currently cueing object (e.g., cartoon figureleft food well and egg-right food well). Displacing a correct washer allowed the rat to retrieve a piece of cereal reward from the food well, whereas displacing a wrong one terminated the trial without further correction. Rats were pretrained in this task to criterion ( $\geq 75 \%$ correct for both conditions for two consecutive days) before receiving bilateral cannula implantations in the dorsal hippocampus.

\section{Histological verification of cannula positions}

The locations of injection cannula tips were verified histologically under the microscope (Fig. 2) after all experiments were finished. Three animals were excluded from final analysis due to misplacements of cannulae, and the remaining 17 rats all showed the cannula tip positions within the dorsal hippocampi.

\section{Hippocampus is necessary for cued retrieval of paired associates in the absence of delay}

After surgery, rats were retrained to criterion, and a drug injection schedule began afterward. First, the rat was injected with phosphate-buffered saline (SAL) in the hippocampus for $2 \mathrm{~d}$. Rats performed well above $80 \%$ correct in the task with SAL infusions for two consecutive days (blocked as SAL1 as shown in Fig. 3A) with minimal variance, as they did during pre-drug conditions. However, the same rats were severely impaired when muscimol (MUS) $(0.5 \mu \mathrm{g} / 0.5 \mu \mathrm{L})$, a GABA-A receptor agonist, was injected for four consecutive days in the hippocampus, showing barely above chance-level performance (MUS1 and MUS2 in Fig. 3A). Two days of SAL injections afterward (SAL2 in Fig. 3A) made the rats regain their normal performance. A repeated measures ANOVA showed a highly significant effect of drug condition $\left(F_{(4,32)}=60.1, P<0.001\right)$. All possible paired comparisons between SAL and MUS conditions showed significant differences ( $P$ 's $<0.001)$. Post-hoc paired comparisons between the pre-injection and two SAL conditions or between the two MUS conditions did not result in significant differences $(P$ 's $>0.05)$. The latency measured between the start box exit and object choice was not significantly different between SAL and MUS conditions $\left(t_{(8)}<1\right.$, $n . s . ;$ paired $t$-test) (Fig. 3B). No innate preference or avoidance for a particular object was seen in this task, because no significant bias was detected toward a particular object-related food well in the first 10 trials of the first day of training $\left(t_{(8)}<1\right.$, n.s.; one sample $t$-test).

In order to address the possibility that the rats under hippocampal MUS injections might have used a nonhippocampal strategy such as an egocentric response strategy (Packard et al. 1989; Packard and McGaugh 1996), a subset of rats $(n=6)$ was tested in the same task in total darkness (Fig. 3C). This was to test whether the rats were able to perform normally without using allocentric cues in the room. Both SAL and MUS groups performed at chance level (Fig. 3D; 50\% shown as a dotted line) when the room cues were made invisible ( $P$ 's $>0.5$ for both groups; one-sample $t$-test), which suggests strongly that the object-cued paired associate task in this study required allocentric distal cues in the room. No significant difference in performance was found between SAL and MUS groups $\left(t_{(4)}<1\right.$, n.s.; independentsamples $t$-test). We further confirmed that the deficits in performance in the dark were not related to object recognition, because rats were able to discriminate objects normally in total darkness, presumably using other sensory modalities (Supplemental Fig. 1).

The results overall strongly demonstrate that the object-cued retrieval of paired associate memory is severely impaired when the dorsal hippocampus is unavailable, even in the absence of delay, and also when only two familiar paired associates were repeatedly tested without trial uniqueness.

\section{Rats can discriminate objects under hippocampal inactivations}

The deficits observed under MUS in the object-cued paired associate task (Fig. 3A) might be attributable to impairment in recognizing object identity. For testing this alternative hypothesis, we next trained the same rats in a simple discrimination task for the two objects (Fig. 4A) that had been used as cueing objects in the main task. In order to test acquisition, the rats assigned to a SAL

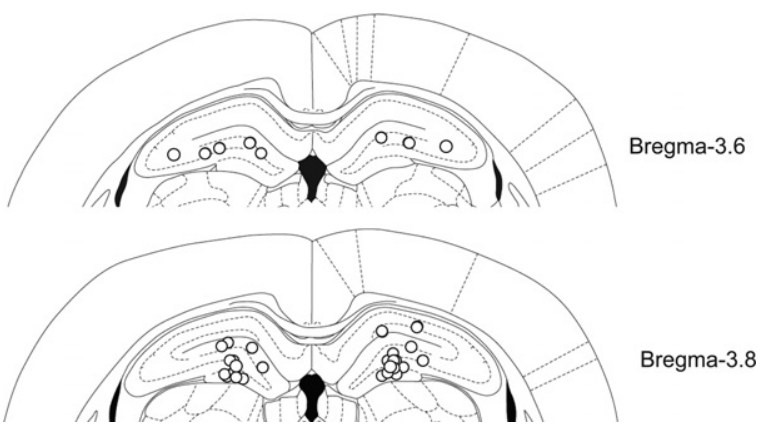

Figure 2. Schematic illustration of the cannula tip positions in the dorsal hippocampus. 

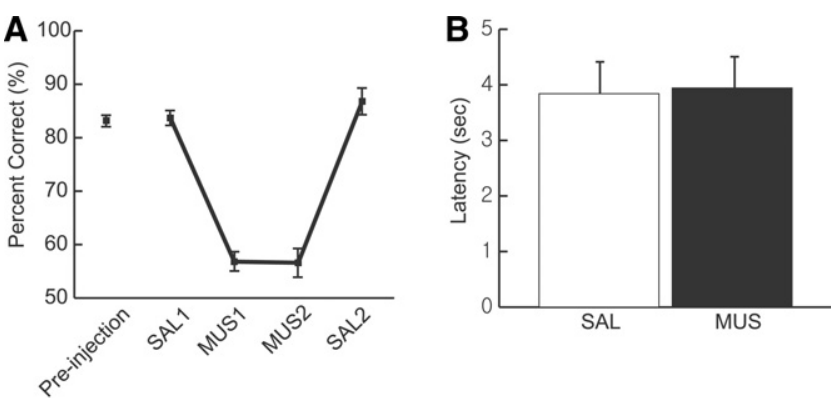

C

D
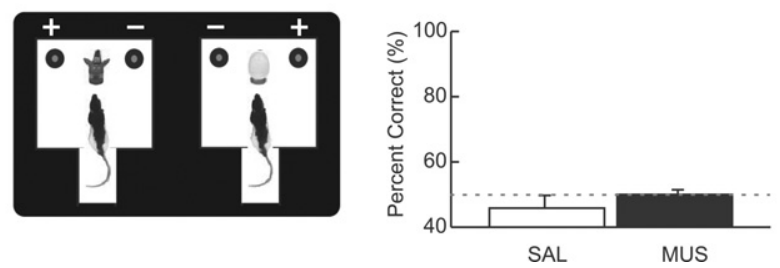

Figure 3. Performance in the object-cued paired associate task. $(A)$ Percent correct was measured for different drug-injection conditions. When saline was infused in the dorsal hippocampus before (SAL1) and after (SAL2) the MUS injections (MUS1 and MUS2), performances were comparable to the condition in which no solution was infused (preinjection). However, once the dorsal hippocampus was inactivated (MUS1 and MUS2), the performance dropped to near chance level, and these were significantly different from SAL conditions. Mean \pm S.E.M. (B) Latency measure for SAL and MUS conditions. There was no significant difference between SAL and MUS conditions (SAL1 and SAL2 were combined to SAL, and MUS1 and MUS2 were merged to MUS). Mean \pm SEM. (C) Control experiments for object-cued paired associate task in total darkness. The rat was required to choose the correct reward location (denoted by + ) using the cueing object in complete darkness. An infrared video camera (SONY Handycam) was used to record the behavioral session and helped the experimenter in the dark environment. Rats were randomly assigned to either the saline (SAL) or muscimol (MUS) injection group. $(D)$ Behavioral performance during the object-cued paired associate task in the dark. Both SAL and MUS groups performed at chance level (50\% shown as a dotted line) when the room cues were made invisible. Mean \pm SEM.

group were trained with SAL infusions in the hippocampus, and a MUS group was trained in the same task with MUS injections prior to each training session until the rats learned to criterion $(\geq 75 \%$ correct for $2 \mathrm{~d}$ in a row). As demonstrated in prior studies (Forwood et al. 2005; Lee and Solivan 2008, 2010), hippocampus is not involved in this type of simple object discrimination, and the results further supported our prior observations. As shown in Figure 4B, both groups reached criterion in $\sim 3 \mathrm{~d}$ (SAL $3.4 \pm$ 0.5 , MUS $3.3 \pm 0.3$; mean \pm SEM) and no significant difference was found between the two groups $\left(t_{(7)}<1\right.$, n.s.; independent samples $t$-test). No innate bias toward a particular object was found when the first five trials on the first training day were examined $\left(t_{(7)}<1, n\right.$.s.; one sample $t$-test).

Since the rats newly learned the object discrimination task in the above manipulations (Fig. 4B), as opposed to being tested for retrieval in the main task (Fig. 3A), we additionally trained an independent group of rats $(n=8)$ to group. Mean \pm SEM. criterion (object-food well associations counterbalanced), implanted cannulae in the dorsal hippocampus, and then tested for the retrieval of the task by using a similar drug-injection protocol used in the main task (i.e., SAL-MUS-MUS-SAL). As shown in Figure 4C, there was no impairment in performance with MUS inactivations, and the performance actually steadily increased as days passed $\left(t_{(14)}<1, n\right.$.s.; independent-samples $t$-test). The results from the control experiments strongly suggest that, when injected with MUS in the hippocampus during the object-cued retrieval of paired associate memory (Fig. $3 \mathrm{~A}$ ), rats were able to recognize the identity of the cueing object.

\section{Rats were able to remember fixed spatial locations under hippocampal inactivations}

Since the rats were able to discriminate the objects under MUS conditions (Fig. 4B,C; Supplemental Fig. 1), the impairment in performance in the main task (Fig. 3A) could be related to their inability to discriminate the two food-well locations in the choice platform when injected with MUS. In order to examine this possibility, we tested the same rats in a spatial reference memory task in the same apparatus (Fig. 5A). Specifically, no object cueing was involved in this task and the rats were simply required to learn a fixed spatial position (between the two food wells covered with identical washers) associated with reward in a given session. Both food wells contained rewards for the first trial in a session (which was not counted for analysis) and the food-well position opposite to the first choice was constantly rewarded in the remaining trials in that session. As in the simple object discrimination task, rats were trained under either SAL or MUS condition until they reached criterion performance. One of the rats was dropped after the simple object discrimination task due to health issues, and the data were analyzed based on seven rats. We found no significant difference in acquisition between the drug groups (SAL 2.2 \pm 0.2 , MUS $2.3 \pm 0.3$; mean \pm SEM) (Fig. 5B) in acquiring this spatial reference memory task $\left(t_{(7)}<1\right.$, n.s.; independentsamples $t$-test). The same results were obtained when a different group of rats $(n=7)$ was pretrained in the spatial reference memory task and tested for retrieval under hippocampal MUS inactivations $\left(t_{(12)}<1, n\right.$.s.; independent-samplest-test) (Fig. 5C). No significant innate bias toward a certain location was detected when the rats first experienced the task (the first five trials on the first day of training; $t_{(6)}<1, n$.s.; one sample $t$-test). These results suggest that the performance deficits in MUS-inactivated rats in the object-cued paired associate task (Fig. 3A) may not be solely attributable to the impairment in discriminating the two locations.
A

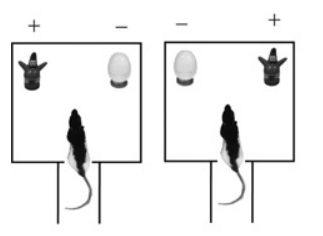

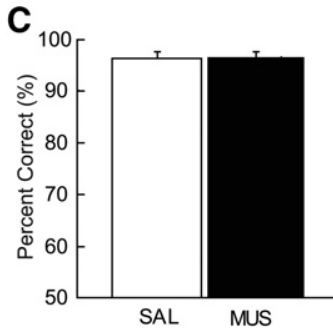

Figure 4. Simple object discrimination task. (A) The objects previously used as cues were presented at the rewarding places in the choice platform. Only choosing the cartoon figure was rewarded (denoted by + ). (B) Total number of days to performance criterion ( $\geq 75 \%$ correct for $2 \mathrm{~d}$ in a row in both conditions) for learning simple object discrimination. Both groups required similar amounts of training to criterion. Mean \pm SEM. (C) Performance in the simple object discrimination task under SAL and MUS injections in the hippocampus. There was no significant difference between SAL group and MUS 
A

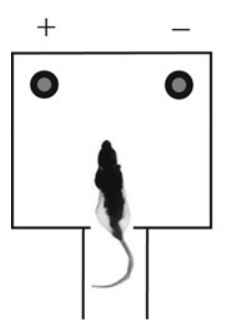

B

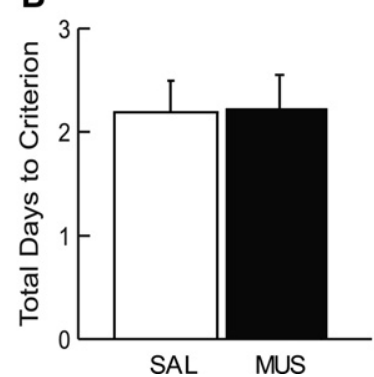

C

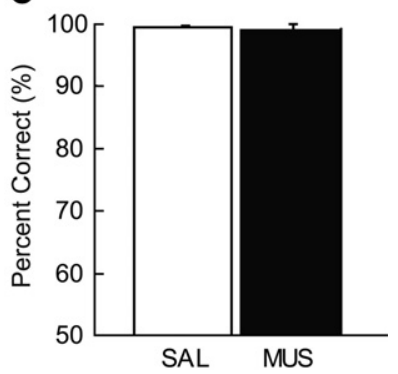

Figure 5. Simple spatial discrimination task. $(A)$ Object-cued retrieval was not necessary and the rat was simply required to choose a fixed reward location predetermined for a given session to obtain reward (left food well in this example). (B) Both SAL and MUS groups were tested to criterion $(>75 \%$ correct for $2 \mathrm{~d}$ in a row in both conditions) in acquisition of simple spatial discrimination task. There was no significant difference between SAL and MUS groups. Mean \pm SEM. (C) Performance in the same task for SAL and MUS groups. Both groups performed almost perfectly in the task and there was no significant difference between the two groups. Mean \pm SEM.

\section{Discussion}

In the current study, rats learned to choose one of the two locations according to the identity of a cueing object in between the locations. Since the cueing object and its spatial paired associate candidates were presented simultaneously in this study, there was no need for maintaining the cueing item information during delay. Moreover, the object-place paired associates were highly familiar to the rats by the time they were tested. In this experimental setting with seemingly reduced cognitive load, however, rats were severely disrupted in performing this task with dorsal hippocampal inactivations. Our control experiments suggest that the rats were able to discriminate the cueing objects from each other and also knew the difference between the two spatial locations without paired associative demand when the dorsal hippocampus was inactivated. Therefore, the results strongly suggest that retrieving the associative link itself between a nonspatial cue and its paired associate location was impaired with hippocampal inactivations, and suggest that other additional cognitive requirements such as working memory demand during delay and onetrial learning condition may not be necessary when testing the hippocampal role in paired associate memory.

In the current study, the scope of discussion is restricted to paired associate memory based on cued retrieval paradigms only. Specifically, putting a temporal gap between sample and test phases in a delayed-cued retrieval paradigm in animals (Day et al. 2003; Tse et al. 2007; Kesner et al. 2008) creates a retrieval condition closer to "recall" as opposed to "recognition" in human experiments (Duzel et al. 2001; Small et al. 2001; Meltzer and Constable 2005) as the critical information (i.e., cue for the to-be-retrieved paired associate) is absent at the time of memory retrieval. The cued retrieval paradigm is used because it is difficult to teach rats a true recall test due to the lack of verbal communication that can be used to convey the rule, as in human studies. Otherwise, animal models have adopted mostly a recognition test in which candidate targets composed of correct and foil paired associates are given and the animal is required to make a choice among the candidates (Staubli et al. 1995; Bunsey and Eichenbaum 1996; Kesner et al. 2005, 2008; Lee and Solivan 2008, 2010; Talpos et al. 2009). Paired associate task demands were critical in most of these recognition and recall tasks, and prior studies showed the importance of the hippocampus in paired associate memory (Gilbert and Kesner 2002, 2003; Day et al. 2003; Eacott and Norman 2004; Rajji et al. 2006; Lee and Solivan 2008, 2010; Talpos et al. 2009). Since the objects were never directly presented at their paired associate locations in the 2006; Kesner et al. 2008) and several studies have shown that the hippocampus is indeed critical for object-place paired association (Gilbert and Kesner 2002, 2003; Day et al. 2003; Eacott and Norman 2004; Rajji et al. 2006; Talpos et al. 2009; Barker and Warburton 2011). The novelty of our study comes from demonstrating for the first time that one of the key ingredients, i.e., delay, taken for granted for a long time in testing hippocampal functions in animal models (Deadwyler et al. 1996; Young et al. 1997; Gilbert et al. 1998; Steele and Morris 1999; Morris 2001; Day et al. 2003; Lee and Kesner 2003a,b; Schon et al. 2008) may not be a necessary component, especially for testing its function in paired associate memory. Notably, the delayed-cued paired associate tasks recently used for showing the role of hippocampus in paired associate memory (Day et al. 2003; Tse et al. 2007; Kesner et al. 2008) contained both delay and paired associative components. The rats in those tasks were cued by a sample in the start box, and left the sampled cue behind as they exited the start box to look for the cued paired associate location. Lesions or perturbations in the hippocampus impaired the rat's performance (Day et al. 2003; Tse et al. 2007; Kesner et al. 2008). In all of these tasks, however, rats must navigate in a large open space upon leaving the start box, which should certainly make the maintenance of the cued item in memory more challenging because of the interference between navigational and working memory demands. We reasoned that removing the temporal gap between the cue presentation and the choice of its paired associate would test the paired associative function of the hippocampus more directly. The results from our study suggest that a cued recall task involving neither delay nor navigation is still an important paradigm that can be used for testing the function of the hippocampus in rodents.

The results from our control experiments demonstrate that simple object discrimination or spatial discrimination may not depend on the hippocampus. Interpreting the results from the simple object discrimination experiment (Fig. 4) is relatively straightforward in light of the literature (Mumby et al. 1999; Lee and Solivan 2008; Abe et al. 2009; Jo and Lee 2010), whereas it requires some speculation as to why the simple spatial discrimination task (Fig. 5) was not dependent on the hippocampus when the cued retrieval requirement was absent. First, it is worth mentioning that retrieving well-established spatial reference memory (i.e., remembering a fixed location in association with reward) is less dependent on the hippocampal circuits, compared with working memory tasks in which multiple candidate target locations must be dynamically associated and disassociated with reward (McNaughton et al. 1986; Morris 1989; Jarrard 1993; Schmitt 
et al. 2003; Niewoehner et al. 2007). The simple spatial discrimination task (Fig. 5) in our study may be considered as a spatial reference memory task in this regard. Second, it is possible that the rats used an allocentric strategy in the object-cued paired associate task (Fig. 3), but switched to an egocentric strategy (Packard et al. 1989; Packard and McGaugh 1996) when simply required to discriminate the food wells on its left vs. right side in the absence of the cueing object (Fig. 5). In support of the usage of allocentric cues in the main task, both SAL and MUS groups performed at chance level when they were tested in total darkness for the object-cued paired associate task (Fig. 3D). If the rats were able to adopt the response strategy (as they might have in the simple visual discrimination task), the animals should have performed well in the dark environment also. However, the fact that the rats could not perform normally at all in the dark illustrates that the object-cued paired associate task required the visual cues in the surrounding environment. The possibility that the rats were unable to identify cueing objects in the dark is unlikely, because a subset of rats that we tested in a simple object discrimination task in the dark had no problem with discriminating the two objects without using vision (presumably using other sensory modalities; Supplemental Fig. 1A,B). Furthermore, we observed that the cueing object in between the two food wells served as a powerful behavioral controller, because the rat paused in front of the cueing object before making a choice. The rat took time in front of the cueing object and appeared to process the cueing object and its background environment before making a choice in the object-cued paired associate task, whereas the absence of this pause (thus continuous run toward a food well) in the simple spatial discrimination task may have biased the rats to use an egocentric strategy more (Pearce et al. 1998; Holdstock et al. 2000). In summary, although the results from the simple spatial discrimination task may not be able to directly address the issue of whether spatial strategy was used in the main task, it is certainly clear that the rats could have used a nonhippocampal strategy in the object-cued paired associate task (as they possibly did in the simple spatial discrimination task) under MUS in hippocampus, but they failed to do so (Fig. 3B,D). Overall, the results support that object-place paired associate memory with great dependence on distal visual cues was tested in the main task.

It is important to emphasize also that the paired associates involved familiar objects and places in the current study, which was far from being trial unique compared with other studies (Day et al. 2003; Kesner et al. 2008). Nonetheless, robust performance deficits were observed when MUS was infused in the hippocampus. This may relate to the hippocampal functions in disambiguating overlapping spatial representations (Gilbert et al. 1998; Tanila 1999; Rolls and Kesner 2006; Leutgeb et al. 2007; McHugh et al. 2007). We speculate that the hippocampal function of retrieving orthogonal representations of object-place paired associates were significantly compromised with MUS inactivations in the hippocampus in the current study. Control rats typically paused in front of the cueing object in our study before making a choice for either spatial location. It is possible that at the time of sampling the cueing object, the hippocampus processed the object and its background spatial context as an objectembedded scene as in a scene discrimination task (Gaffan 1994; Gaffan and Parker 1996; Murray et al. 1998; Rolls and Xiang 2005). If that was the case, the discrimination must have been subtle, because all visual cues in the room were maintained constant across trials. We used prominent visual cues (Fig. 1A) in the testing room to encourage an allocentric strategy and the control experiment performed in total darkness (Fig. 3D) supports the rationale of using such manipulations by showing that the rats in our study were incapable of making spatial choices in the absence of distal visual cues. Such requirement for maximal pattern separation in the hippocampus (O'Reilly and McClelland 1994; Kesner et al. 2004; Leutgeb et al. 2007; McHugh et al. 2007) between the paired associate conditions may underlie the striking impairment with hippocampal inactivations in the current study.

The paired associate memory paradigm has been extensively used in human and animal models for its known dependence on the hippocampus (Bunsey and Eichenbaum 1993, 1996; Cho and Kesner 1995; Vargha-Khadem et al. 1997; Gilbert and Kesner 2002; Day et al. 2003; Kesner et al. 2005; Tse et al. 2007; Hannula and Ranganath 2008; Lee and Solivan 2008, 2010; Holdstock et al. 2010; Lee and Kim 2010). A one-trial delayed-cued retrieval task for paired associate memory (Day et al. 2003; Kesner et al. 2008) may certainly contribute to the investigation of the role of hippocampus in episodic-like memory in animals. However, since a single behavioral task may require a host of disparate cognitive processes at the same time, a behavioral task may benefit from keeping the required cognitive processes to its necessary minimum if the goals are to elucidate detailed mechanisms. In our study, we have demonstrated that the hippocampal function in object-cued paired associate memory can be studied effectively in a surprisingly simple situation if necessary conditions are met.

\section{Materials and Methods}

\section{Subjects}

Twenty male Long-Evans rats (270-330 g) were used in the study. Three of them were removed from analysis due to cannula misplacements, leaving 17 rats available for final data analysis. Each rat was housed in a standard rodent cage and maintained on a 12-h light/dark cycle, and all of the experimental sessions were conducted during the light phase. The rat's weight was maintained at $80 \%$ of its free-feeding weight. Water was provided ad libitum. All experimental and surgical protocols conformed to the NIH Guide for the Care and Use of Laboratory Animals and the Institutional Animal Care and Use Committee of the Seoul National University.

\section{Behavioral apparatus}

An elevated linear track ( $42 \mathrm{~cm}$ in length and $8 \mathrm{~cm}$ in width) made of Plexiglas (Fig. 1A) was used throughout the experiments. A start box $(15 \times 23 \times 34 \mathrm{~cm})$ with an opaque guillotine door was located at one end of the track. Attached to the other end of the track was a rectangular choice platform $(24 \times 25.5 \mathrm{~cm})$, in which three food wells (left, center, and right food wells) were aligned linearly. Each food well was $2.5 \mathrm{~cm}$ in diameter and $0.5 \mathrm{~cm}$ in depth. They were $5.5-\mathrm{cm}$ apart from each other. Either the left or right food well was used for hiding a reward. Both food wells were always covered with identical, black metal discs. The linear track was placed in the center of a circular curtained area and multiple distinctive cues were available along the curtains as distal visual cues (Fig. 1A). A halogen light was placed in the center of the ceiling next to a digital camera. The illumination level was maintained at $200 \mathrm{~lx}$ (when measured from the center of the linear track). Loudspeakers were placed behind the curtains to provide white noise during the behavioral experiment, and the volume was maintained at $70 \mathrm{~dB}$. Two objects used as cue objects were an egg-shaped junk toy and a cartoon figure of similar size (Fig. 1B).

\section{Handling, familiarization, and shaping}

Once naive rats arrived, they were housed individually during a week of acclimation period. Then, handling started and the rats were accustomed to eating sugarcoated cereal (Froot Loops, Kellogg's). The rat was handled by an experimenter for the first $5 \mathrm{~min}$ and was put on the surface of a laboratory cart for the next $10 \mathrm{~min}$, with several pieces of cereal spread on the surface of the cart. If the rat ate $>10$ pieces of cereal in $15 \mathrm{~min}$, the animal was introduced to the behavioral testing room for familiarization. The illumination level, white noise level, visual cues, and other 
environmental factors were maintained the same throughout the experiment. During the initial phase of the familiarization $(\sim 2 \mathrm{~d})$, each rat was placed in the start box, and when its door was opened, the rat explored the linear track where cereal rewards were randomly scattered.

Once the rat started to move and eat cereal comfortably in the track for 15 min without any urination and defecation, a more specific way of retrieving food was taught. Specifically, a half piece of cereal was given in the center food well, and a black metal disc covered the food well. When the experimenter opened the start box door, the rat came out and ran along the linear track for retrieving the cereal reward by displacing the disc. After the animal retrieved the cereal from the food well, the experimenter gently guided the animal back to the start box so that it consumed the food reward in the start box. After a few days of training, rats returned to the start box voluntarily for consuming the cereal reward. Once the rat was able to repeat this food-retrieving routine for 32 times within $30 \mathrm{~min}$, the animal was considered ready for the object-cued paired associate task.

\section{Presurgical training}

In the object-cued paired associate task (Fig. 1B), the rat ran along the linear track when the door of the start box was opened and reached the choice platform at the end of the track. The left and right food wells in the choice platform were covered with identical metal discs and a toy object (either an egg-shaped plastic toy and a cartoon figure) was placed just in front of the center food well positioned between the left and right food wells. This arrangement allowed the rats to sample the cueing object first before making a choice between the two food wells. Food reward was hidden in either the left or right food well, and the identity of the cueing object determined which food well contained the reward. For example, if the egg-shaped toy was presented as a cue, the rat was required to displace the disc in the right food well to obtain a reward and vice versa when the cartoon figure was introduced. Both objects appeared in equal numbers of trials throughout a 32 -trial session. Once the rat was trained to criterion ( $\geq 75 \%$ correct for both egg-right and cartoon figure-left conditions for two consecutive days), it received surgery for bilateral cannula implantation in the dorsal hippocampus.

\section{Surgery}

Bilateral guide cannulae (26G; Plastics One) coupled with stylets (33G, $1 \mathrm{~mm}$ protrusion from the tip of the guide cannula) were implanted in the hippocampus. Detailed surgical procedures were as follows. Each animal was deeply anesthetized with isoflurane in an induction chamber. The animal was then injected with Nembutal $(70 \mathrm{mg} / \mathrm{kg})$ intraperiotoneally and placed in a stereotaxic instrument (Stoelting), and an incision was made along the midline of the scalp. The anesthesia was maintained by isoflurane afterward throughout surgery. The skull was exposed and the instrument was adjusted to ensure a flat skull surface. Small burr holes were drilled in the skull and the following coordinates were used for cannula implantations: $3.8 \mathrm{~mm}$ posterior to bregma, $2.6 \mathrm{~mm}$ lateral to midline, and $4.0 \mathrm{~mm}$ ventral from the skull surface. Small burr holes were drilled in the periphery of the skull for positioning jeweler's screws $(0-80)$. After the insertion of the bilateral cannulae in the hippocampus, bone cement (Zimmer) was used around the skull screws and the cannulae to ensure firm implantation. All protocols conformed to the NIH Guide for the Care and Use of Laboratory Animals and the Institutional Animal Care and Use Committee at the Seoul National University.

\section{Postsurgical behavioral testing and control experiments}

After a week of recovery from surgery, the rat was retrained to criterion before a drug injection schedule began. Muscimol (MUS) $(0.5 \mu \mathrm{g} / 0.5 \mu \mathrm{L})$, a GABA-A receptor agonist, was used to temporally inactivate the dorsal hippocampus. The rat was injected with SAL for first $2 \mathrm{~d}$, followed by $4 \mathrm{~d}$ of MUS injections. Then, the injection schedule completed with two more days of SAL injections. On drug-injection days for behavioral testing, the rat was first anesthetized lightly with isoflurane. While the rat was maintained at a low dose of isoflurane anesthesia through a portable nose mask, dummy stylets (33G) were removed and an injection cannula (33G) extending $1 \mathrm{~mm}$ below the tip of the guide cannula (26G) was inserted. The injection cannula was backfilled beforehand with mineral oil and connected to a $10-\mu \mathrm{L}$ syringe (Hamilton). Either SAL or MUS was injected at a rate of $10 \mu \mathrm{L} / \mathrm{h}$ using a microinfusion pump (KD Scientific). The injection quantity was $0.5 \mu \mathrm{L} /$ hemisphere. The injection cannula was left in place for an additional $60 \mathrm{sec}$ before and after the drug injection to allow a proper diffusion of the drug from its tip. The rat was then returned to its home cage and behavioral testing began in $20 \mathrm{~min}$.

After the rat finished the object-cued paired associate task with the above drug schedule for $8 \mathrm{~d}$, the following series of control experiments were performed to examine some alternative hypotheses: (1) the same task in complete darkness, (2) simple object discrimination, (3) simple spatial discrimination, and (4) simple object discrimination in the dark.

\section{Object-cued paired associate task in the dark}

In this control experiment, we sought to test whether the rats used visual cues in the object-cued paired associate task; everything remained the same as in the main task, except that the rats performed the task in complete darkness. An infrared video camera (SONY Handycam) was used to record the behavioral session and helped the experimenter in the dark environment.

\section{Simple object discrimination}

In a simple object-discrimination task, we tested the possibility of perceptual impairment under hippocampal MUS inactivation in recognizing a cueing object. In this task, the rat ran along the linear track from the start box and found two objects (the same objects used as cues during the main task) overlying the left and right food wells in the choice platform at the same time. Both objects switched their positions between left and right food wells pseudorandomly in a counterbalanced manner during a session. Rats were trained to displace the rewarded object, the cartoon figure, over the unrewarded figure, the egg-shaped object under drug infusion (MUS or SAL), until it reached criterion ( $\geq 75 \%$ correct for $2 \mathrm{~d}$ in a row) for testing acquisition $(n=9)$. An independent group $(n=8)$ of rats were also tested for retention of the task.

\section{Simple spatial discrimination}

If the rat was unable to discriminate the two spatial locations in the main task, performance deficit was expected regardless of the hippocampal associative function. A simple spatial discrimination task was carried out to test this. In this task, no object was presented except for the two metal discs covering the food wells. For the first trial, there was no food given under any disc and the rat made an arbitrary choice. Afterward, the food well that was not chosen by the rat in the first trial was rewarded throughout the session. Nine rats were used for testing acquisition and another seven rats were used for testing retention of the task.

\section{Simple object discrimination in dark}

One way of interpreting the impairment in performance in control experiment is that the rat could not sample visual identity information of the cueing object when it was dark. To test this possibility, all procedures were maintained the same as in the simple object discrimination task above, except that the task was carried out in total darkness. Six rats were assigned to the object-cued paired associate task in dark.

\section{Histology}

Histological verifications of cannula positions were performed soon after the completion of the control experiments. Rats were exposed to a lethal dose of $\mathrm{CO}_{2}$ gas. A transcardial perfusion was performed afterward with $0.9 \%$ saline and $4 \%$ formaldehyde solution. The brain was extracted and stored in a 4\% formalin-30\% sucrose solution at $4^{\circ} \mathrm{C}$ for $72 \mathrm{~h}$. The brain was then embedded 
in sucrose gel, frozen, and cut in coronal sections $(40 \mu \mathrm{m})$ on a sliding microtome (Microm, Thermo Fisher Scientific). Sections were later Nissl stained with thionin (Sigma-Aldrich) and were photomicrographed for the verification of cannula positions.

\section{Acknowledgments}

The current study was supported by the World Class University program of the Korea Science and Engineering Foundation funded by the Ministry of Education, Science and Technology (R31-10089) and by NIMH RO1 MH079971. We thank Jihye Lee and Dayun Jeong for assistance in data collection.

\section{References}

Abe H, Ishida Y, Nonaka H, Iwasaki T. 2009. Functional difference between rat perirhinal cortex and hippocampus in object and place discrimination tasks. Behav Brain Res 197: 388-397.

Barker GRI, Warburton EC. 2011. When Is the hippocampus involved in recognition memory? J Neurosci 31: 10721-10731.

Bennett MR, Gibson WG, Robinson J. 1994. Dynamics of the CA3 pyramidal neuron autoassociative memory network in the hippocampus. Philos Trans R Soc Lond B Biol Sci 343: 167-187.

Bunsey M, Eichenbaum H. 1993. Critical role of the parahippocampal region for paired-associate learning in rats. Behav Neurosci 107: 740-747.

Bunsey M, Eichenbaum H. 1996. Conservation of hippocampal memory function in rats and humans. Nature 379: 255-257.

Cho YH, Kesner RP. 1995. Relational object association learning in rats with hippocampal lesions. Behav Brain Res 67: 91-98.

Day M, Langston R, Morris RGM. 2003. Glutamate-receptor-mediated encoding and retrieval of paired-associate learning. Nature 424: 205-209.

Deadwyler SA, Bunn T, Hampson RE. 1996. Hippocampal ensemble activity during spatial delayed-nonmatch-to-sample performance in rats. J Neurosci 16: $354-372$.

Duzel E, Vargha-Khadem F, Heinze HJ, Mishkin M. 2001. Brain activity evidence for recognition without recollection after early hippocampal damage. Proc Natl Acad Sci 98: 8101-8106.

Eacott MJ, Norman G. 2004. Integrated memory for object, place, and context in rats: A possible model of episodic-like memory? J Neurosci 24: $1948-1953$

Forwood SE, Winters BD, Bussey TJ. 2005. Hippocampal lesions that abolish spatial maze performance spare object recognition memory at delays of up to 48 hours. Hippocampus 15: 347-355.

Gaffan D. 1994. Scene-specific memory for objects: A model of episodic memory impairment in monkeys with fornix transection. J Cogn Neurosci 6: $305-320$.

Gaffan D, Parker A. 1996. Interaction of perirhinal cortex with the fornix-fimbria: Memory for objects and "object-in-place" memory. I Neurosci 16: $5864-5869$

Gilbert PE, Kesner RP. 2002. Role of the rodent hippocampus in paired-associate learning involving associations between a stimulus and a spatial location. Behav Neurosci 116: 63-71.

Gilbert PE, Kesner RP. 2003. Localization of function within the dorsal hippocampus: The role of the CA3 subregion in paired-associate learning. Behav Neurosci 117: 1385-1394.

Gilbert PE, Kesner RP, DeCoteau WE. 1998. Memory for spatial location: Role of the hippocampus in mediating spatial pattern separation. J Neurosci 18: 804-810.

Hannula DE, Ranganath C. 2008. Medial temporal lobe activity predicts successful relational memory binding. J Neurosci 28: 116-124.

Hasselmo ME, McClelland JL. 1999. Neural models of memory. Curr Opin Neurobiol 9: 184-188.

Holdstock JS, Mayes AR, Cezayirli E, Isaac CL, Aggleton JP, Roberts N. 2000. A comparison of egocentric and allocentric spatial memory in a patient with selective hippocampal damage. Neuropsychologia 38: 410-425.

Holdstock JS, Crane J, Bachorowski JA, Milner B. 2010. Equivalent activation of the hippocampus by face-face and face-laugh paired associate learning and recognition. Neuropsychologia 48: 3757-3771.

Jarrard LE. 1993. On the role of the hippocampus in learning and memory in the rat. Behav Neural Biol 60: 9-26.

Jo YS, Lee I. 2010. Disconnection of the hippocampal-perirhinal cortical circuits severely disrupts object-place paired associative memory. $J$ Neurosci 30: 9850-9858.

Kesner RP, Lee I, Gilbert P. 2004. A behavioral assessment of hippocampal function based on a subregional analysis. Rev Neurosci 15: 333-351.

Kesner RP, Hunsaker MR, Gilbert PE. 2005. The role of CA1 in the acquisition of an object-trace-odor paired associate task. Behav Neurosci 119: $781-786$.
Kesner RP, Hunsaker MR, Warthen MW. 2008. The CA3 subregion of the hippocampus is critical for episodic memory processing by means of relational encoding in rats. Behav Neurosci 122: 1217-1225.

Kim JJ, Fanselow MS. 1992. Modality-specific retrograde amnesia of fear. Science 256: 675-677.

Lee I, Kesner RP. 2003a. Differential roles of dorsal hippocampal subregions in spatial working memory with short versus intermediate delay. Behav Neurosci 117: 1044-1053.

Lee I, Kesner RP. 2003b. Time-dependent relationship between the dorsal hippocampus and the prefrontal cortex in spatial memory. J Neurosci 23: $1517-1523$.

Lee I, Kim J. 2010. The shift from a response strategy to object-in-place strategy during learning is accompanied by a matching shift in neural firing correlates in the hippocampus. Learn Mem 17: 381-393.

Lee I, Solivan F. 2008. The roles of the medial prefrontal cortex and hippocampus in a spatial paired-association task. Learn Mem 15: $357-367$.

Lee I, Solivan F. 2010. Dentate gyrus is necessary for disambiguating similar object-place representations. Learn Mem 17: 252-258.

Leutgeb JK, Leutgeb S, Moser M-B, Moser EI. 2007. Pattern separation in the dentate gyrus and CA3 of the hippocampus. Science 315: 961-966.

McHugh TJ, Jones MW, Quinn JJ, Balthasar N, Coppari R, Elmquist JK, Lowell BB, Fanselow MS, Wilson MA, Tonegawa S. 2007. Dentate gyrus nmda receptors mediate rapid pattern separation in the hippocampal network. Science 317: 94-99.

McNaughton BL, Barnes CA, Rao G, Baldwin J, Rasmussen M. 1986. Long-term enhancement of hippocampal synaptic transmission and the acquisition of spatial information. J Neurosci 6: 563-571.

Meltzer JA, Constable RT. 2005. Activation of human hippocampal formation reflects success in both encoding and cued recall of paired associates. Neuroimage 24: 384-397.

Morris R. 1989. Synaptic plasticity and learning: Selective impairment of learning rats and blockade of long-term potentiation in vivo by the N-methyl-D- aspartate receptor antagonist AP5. J Neurosci 9: 3040-3057.

Morris RG. 2001. Episodic-like memory in animals: Psychological criteria, neural mechanisms and the value of episodic-like tasks to investigate animal models of neurodegenerative disease. Philos Trans $R$ Soc Lond $B$ Biol Sci 356: 1453-1465.

Mumby DG, Astur RS, Weisend MP, Sutherland RJ. 1999. Retrograde amnesia and selective damage to the hippocampal formation: Memory for places and object discriminations. Behav Brain Res 106: 97-107.

Murray EA, Baxter MG, Gaffan D. 1998. Monkeys with rhinal cortex damage or neurotoxic hippocampal lesions are impaired on spatial scene learning and object reversals. Behav Neurosci 112: 1291-1303.

Niewoehner B, Single FN, Hvalby O, Jensen V, Meyer Zum Alten Borgloh S, Seeburg PH, Rawlins JN, Sprengel R, Bannerman DM. 2007. Impaired spatial working memory but spared spatial reference memory following functional loss of NMDA receptors in the dentate gyrus. Eur J Neurosci 25: $837-846$.

O'Reilly RC, McClelland JL. 1994. Hippocampal conjunctive encoding, storage, and recall: Avoiding a trade-off. Hippocampus 4: 661-682.

Packard MG, McGaugh JL. 1996. Inactivation of hippocampus or caudate nucleus with lidocaine differentially affects expression of place and response learning. Neurobiol Learn Mem 65: 65-72.

Packard M, Hirsh R, White N. 1989. Differential effects of fornix and caudate nucleus lesions on two radial maze tasks: Evidence for multiple memory systems. J Neurosci 9: 1465-1472.

Pearce JM, Roberts AD, Good M. 1998. Hippocampal lesions disrupt navigation based on cognitive maps but not heading vectors. Nature 396: $75-77$.

Rajji T, Chapman D, Eichenbaum H, Greene R. 2006. The role of CA3 hippocampal NMDA receptors in paired associate learning. J Neurosci 26: $908-915$

Rempel-Clower NL, Zola SM, Squire LR, Amaral DG. 1996. Three cases of enduring memory impairment after bilateral damage limited to the hippocampal formation. J Neurosci 16: 5233-5255.

Rolls ET, Kesner RP. 2006. A computational theory of hippocampal function, and empirical tests of the theory. Prog Neurobiol 79: $1-48$.

Rolls ET, Xiang JZ. 2005. Reward-spatial view representations and learning in the primate hippocampus. J Neurosci 25: 6167-6174.

Schmitt WB, Deacon RMJ, Seeburg PH, Rawlins JNP, Bannerman DM. 2003. A within-subjects, within-task demonstration of intact spatial reference memory and impaired spatial working memory in glutamate receptor-a-deficient mice. J Neurosci 23: 3953-3959.

Schon K, Tinaz S, Somers DC, Stern CE. 2008. Delayed match to object or place: An event-related fMRI study of short-term stimulus maintenance and the role of stimulus pre-exposure. Neuroimage 39: 857-872.

Small SA, Nava AS, Perera GM, DeLaPaz R, Mayeux R, Stern Y. 2001. Circuit mechanisms underlying memory encoding and retrieval in the long axis of the hippocampal formation. Nat Neurosci 4: 442-449. 
Squire LR, Clark RE, Knowlton BJ. 2001. Retrograde amnesia. Hippocampus 11: $50-55$

Staubli U, Le TT, Lynch G. 1995. Variants of olfactory memory and their dependencies on the hippocampal formation. JNeurosci 15: 1162-1171.

Steele RJ, Morris RG. 1999. Delay-dependent impairment of a matching-to-place task with chronic and intrahippocampal infusion of the NMDA-antagonist D-AP5. Hippocampus 9: 118-136.

Talpos J, Winters B, Dias R, Saksida L, Bussey T. 2009. A novel touchscreen-automated paired-associate learning (PAL) task sensitive to pharmacological manipulation of the hippocampus: A translational rodent model of cognitive impairments in neurodegenerative disease. Psychopharmacology 205: 157-168.

Tanila H. 1999. Hippocampal place cells can develop distinct representations of two visually identical environments. Hippocampus 9: $235-246$.

Tse D, Langston RF, Kakeyama M, Bethus I, Spooner PA, Wood ER, Witter MP, Morris RGM. 2007. Schemas and memory consolidation. Science 316: 76-82.
Vargha-Khadem F, Gadian DG, Watkins KE, Connelly A, Van Paesschen W, Mishkin M. 1997. Differential effects of early hippocampal pathology on episodic and semantic memory. Science 277: 376-380.

Winocur G. 1990. Anterograde and retrograde amnesia in rats with dorsal hippocampal or dorsomedial thalamic lesions. Behav Brain Res 38: $145-154$.

Young BJ, Otto T, Fox GD, Eichenbaum H. 1997. Memory representation within the parahippocampal region. J Neurosci 17: 5183-5195.

Zola-Morgan S, Squire LR. 1990. The primate hippocampal formation: Evidence for a time-limited role in memory storage. Science 250: $288-290$.

Zola-Morgan S, Squire LR, Amaral DG. 1986. Human amnesia and the medial temporal region: Enduring memory impairment following a bilateral lesion limited to field CA1 of the hippocampus. J Neurosci 6: $2950-2967$.

Received October 18, 2011; accepted in revised form November 1, 2011. 


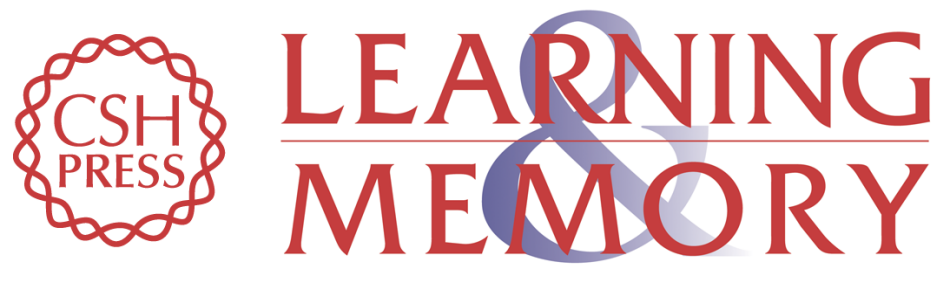

\section{Hippocampus is required for paired associate memory with neither delay nor trial uniqueness}

Jinah Yoon, Yeran Seo, Jangjin Kim, et al.

Learn. Mem. 2012, 19:

Access the most recent version at doi:10.1101//m.024554.111

Supplemental http://learnmem.cshlp.org/content/suppl/2011/12/16/19.1.1.DC1
Material

References This article cites 62 articles, 28 of which can be accessed free at: http://learnmem.cshlp.org/content/19/1/1.full.html\#ref-list-1

License

Email Alerting Receive free email alerts when new articles cite this article - sign up in the box at the Service top right corner of the article or click here. 